\title{
Controlled creation of three-dimensional vortex structures in Bose-Einstein condensates using artificial magnetic fields
}

\author{
James Schloss $\left(1,{ }^{1}\right.$ Peter Barnett $\odot,{ }^{1,2}$ Rashi Sachdeva $\odot,{ }^{1,3}$ and Thomas Busch ${ }^{1}$ \\ ${ }^{1}$ OIST Graduate University, Okinawa 904-0495, Japan \\ ${ }^{2}$ Department of Physics, University of Otago, Dunedin 9054, New Zealand \\ ${ }^{3}$ Mathematical Physics and NanoLund, Lund University, Box 118, 22100 Lund, Sweden
}

(Received 6 October 2019; accepted 30 September 2020; published 23 October 2020)

\begin{abstract}
The physics of quantized vortex excitations in atomic Bose-Einstein condensates has been extensively studied in recent years. Although simple vortex lines are relatively easy to create, control, and measure in experiments, it is a lot more difficult to do the same for vortex ring structures. Here we suggest and explore a method for generating and controlling superfluid vortex rings, vortex ring lattices, and other three-dimensional vortex structures in toroidally trapped superfluid Bose-Einstein condensates by using the artificial magnetic field produced by an optical nanofiber.
\end{abstract}

DOI: 10.1103/PhysRevA.102.043325

\section{INTRODUCTION}

Atomic Bose-Einstein condensates (BECs) are superfluids consisting of neutral, bosonic atoms that have been cooled and condensed into the macroscopic ground state of an external potential [1]. They have been shown to support a large number of flow-related excitations, with the most common ones being quantized vortex lines and vortex rings [2-9]. However, vortices with higher winding numbers are unstable in singly connected condensates, which means that increasing the amount of angular momentum imparted on the superfluid will lead to an increasing number of vortices with a winding number of 1 . These vortex lines interact repulsively and larger numbers will eventually arrange themselves in the form of a triangular, Abrikosov lattice [3], similar to the behavior known for Type II superconductors [10]. Due to the quantization and the homogeneity in winding numbers, single component condensates are often suggested and used for studying superfluid turbulence [11-14].

In a finite-sized atomic condensate without dissipative effects, all vortex lines must have a finite length and either start and end at the cloud surface [2] or reconnect onto themselves [12]. Complex, three-dimensional vortex topologies beyond vortex lines cannot be easily created by stirring or rotating a BEC because the vortex lines generated in this way must follow the axis of rotation; therefore, to consistently control and generate vortex rings or other topological structures, methods beyond stirring are required, and only a small number of experimental realizations of these have been reported $[5,9]$.

Published by the American Physical Society under the terms of the Creative Commons Attribution 4.0 International license. Further distribution of this work must maintain attribution to the author(s) and the published article's title, journal citation, and DOI.
In most cases, including in most theoretical proposals, vortex ring generation in BECs relies on dynamic processes that do not create eigenstates of the system. These include using the decay of dark solitons in multicomponent condensates [5] via the snake instability [15], direct density engineering $[16,17]$, or the collision of symmetric defects [18]. Other theoretical proposals have considered interfering two BECs [19], using spatially dependent Feschbach resonances [20], or direct phase imprinting methods [15]. It should be noted that for inhomogeneously trapped BECs, vortex ring structures are known to be unstable, which has led to difficulties in their experimental observation [21]. In addition, the direct absorption imaging techniques employed in the field of BECs are not well suited to determine whether or not a three-dimensional vortex structure is present in an experimental system, as the known creation mechanisms do not lead to well-defined spatial orientations.

Another method to induce rotational effects in a BEC is through the introduction of artificial magnetic fields, which can be created, for example, by the interaction between an atomic system in a dressed state and an electric field that is tuned near an atomic resonance frequency [22]. In this case, instead of following an axis of rotation, the vortices follow along the artificial magnetic field lines, which allows one to stably generate complex vortex structures by modulating the geometry of the magnetic field profiles.

In this work we will discuss a system that allows for the tunable creation of artificial magnetic fields based on electromagnetic fields that vary strongly over short distances. Such behavior can be found in the near-field regime on the surface of a dielectric system, when light undergoes total internal reflection [23]. For the generation of vortex rings, a suitable dielectric system is the optical nanofiber, which is an optical element that has several propagation modes to allow for the configurable generation of evanescent fields. Nanofiber systems can be created by heating and stretching optical fibers until their thinnest region is roughly hundreds of nanometers 


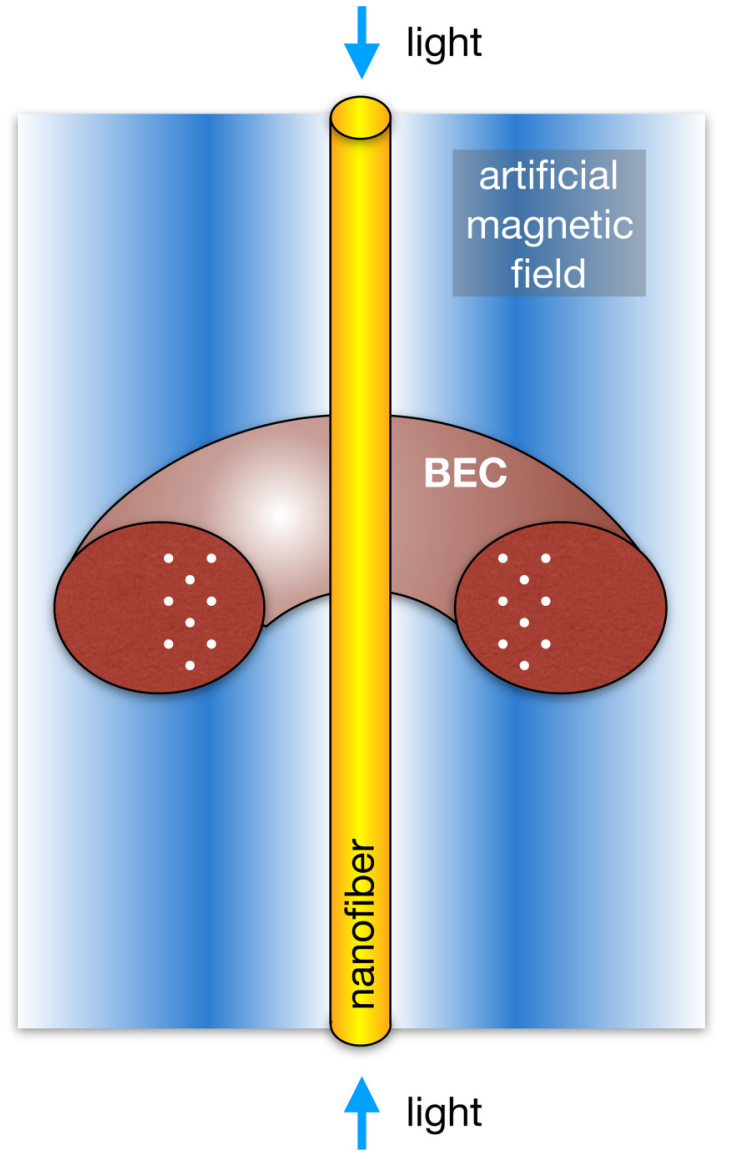

FIG. 1. Schematic of the system. Blue- or red-detuned light is sent into the nanofiber (yellow), creating an evanescent field and artificial magnetic field (blue) that influences the BEC (maroon) held by a toroidal trapping potential. If the artificial magnetic field strength is greater than a threshold value, vortex rings (white) will appear and begin to arrange themselves into a triangular lattice.

in diameter [24,25]. At this scale, the wavelength of light is larger than the diameter of the fiber and the strength of the evanescent field is significantly enhanced [26]. The form of the evanescent field varies significantly depending on the optical modes propagating through the nanofiber, and we will show that this can be used to generate interesting and tunable artificial magnetic fields.

Optical nanofibers are already used in many different experiments with ultracold atoms [27-32], and trapping potentials at around $200 \mathrm{~nm}$ from the fiber surface can be created by using two differently detuned input fields [33,34]. Our proposed setup will allow for the creation of vortex rings in BECs that are trapped toroidally around the fiber at roughly the same distance by coupling the BEC to the evanescent field created by different modes propagating through the nanofiber [35]. A schematic of system is depicted in Fig. 1.

The manuscript is organized as follows: In Sec, II, we will discuss how BECs interact with the evanescent field profiles generated by the optical nanofiber. Then, in Sec. III, we will show simulated results of the vortex configurations that can be generated for different modes and different parameters. In Sec. IV, we conclude and briefly discuss potential extensions of the suggested system.

\section{BOSE-EINSTEIN CONDENSATES IN THE PRESENCE OF AN OPTICAL NANOFIBER}

The superfluid properties of atomic Bose-Einstein condensates are captured by the Gross-Pitaeveskii Equation (GPE), which describes the evolution of the condensate wave function in the mean field limit as [1]

$$
i \hbar \frac{\partial \Psi(\mathbf{r}, t)}{\partial t}=\left[\frac{[p-m \mathbf{A}(\mathbf{r})]^{2}}{2 m}+V(\mathbf{r})+g|\Psi(\mathbf{r}, t)|^{2}\right] \Psi(\mathbf{r}, t) .
$$

Here $p=-i \hbar \frac{\partial}{\partial \mathbf{r}}$ is the standard momentum operator and the kinetic energy term also accounts for the presence of a spatially inhomogeneous artificial vector potential, $\mathbf{A}(\mathbf{r})$. The potential term $V(\mathbf{r})$ describes an external trap and the nonlinear term accounts for the scattering interaction between the atoms. Its strength is given by $g=\frac{4 \pi \hbar^{2} a_{s}}{m}$, where $a_{s}$ is the scattering length of the atomic species and $m$ its mass.

For neutral atomic condensates described by the GPE, the artificial vector potential can take many forms. While in standard magnetism a charged particle acquires an AharonovBohm phase when traveling around a closed contour, for neutral atoms the same can be achieved by creating a situation where the atoms acquires a geometric phase when traveling along such a contour. Berry's phase is an example of such a phase, and in the setup we discuss below it appears as slow atoms travel through a highly inhomogeneous light field and adiabatically follow the dressed eigenstates. When they return to their original position, i.e. when they have traveled along a closed contour, they will have acquired a Berry phase and one can write the corresponding artificial vector potential in terms of Berry's connection as [22]

$$
\mathbf{A}=i \hbar\left\langle\Psi_{l} \mid \nabla \Psi_{l}\right\rangle
$$

where $\Psi_{l}$ is the atomic wave function in some dressed state $l$.

Since we will be considering two-state atoms in the presence of an optical field, the relevant dressed states can be written within the rotating wave approximation as [23]

$$
\begin{gathered}
\left|\Psi_{1}(\mathbf{r})\right\rangle=\left(\begin{array}{c}
\cos [\Phi(\mathbf{r}) / 2] \\
\sin [\Phi(\mathbf{r}) / 2] e^{i \phi(z)}
\end{array}\right), \\
\left|\Psi_{2}(\mathbf{r})\right\rangle=\left(\begin{array}{c}
-\sin [\Phi(\mathbf{r}) / 2] e^{-i \phi(z)} \\
\cos [\Phi(\mathbf{r}) / 2]
\end{array}\right),
\end{gathered}
$$

where $\phi(z)$ is the phase of the optical field and $\Phi(\mathbf{r})=$ $\arctan (|\kappa(\mathbf{r})| / \Delta)$, with $\Delta=\omega_{0}-\omega$ being the detuning and $\kappa(\mathbf{r})=\mathbf{d} \cdot \mathbf{E}(\mathbf{r}) / \hbar$ being the Rabi frequency. The atomic dipole moment is given by $\mathbf{d}$ and $\mathbf{E}(\mathbf{r})$ is the electric field. The condition for adiabatic following of a dressed state is that the gap between the two dressed states should allow for adiabatic elimination of one of them. This gap is given by $\hbar \Omega(r)$, where $\Omega(r)=\sqrt{\Delta^{2}+|\kappa(r)|^{2}}$ is a generalized Rabi frequency, and which leads to a condition for the velocity of the atoms of the form $v \ll \Omega /\left(\left|\left\langle\Psi_{2} \mid \nabla \Psi_{1}\right\rangle\right|\right)$. A detailed analysis of this can be found [36].

The form of the artificial vector potential is therefore determined by the form of the optical fields, and for the nanofiber system it can be controlled by choosing specific optical modes to travel through the fiber. The artificial magnetic field associated with the spatially varying artificial vector potential is 
then given by $\mathbf{B}=\nabla \times \mathbf{A}$, and when the magnetic field lines penetrate the condensates, an artificial Lorentz force will lead to the creation of vortices around these field lines.

To determine which modes will propagate in an optical fiber, one needs to calculate the $V$ number, which is given by $V=k_{0} a \sqrt{n_{1}^{2}-n_{2}^{2}}$. Here $a$ is the fiber radius, $n_{1}$ is the refractive index of the fiber, $n_{2}$ is the refractive index of the cladding, and $k_{0}=\omega / c$ with $\omega$ being the frequency of the input light beam. In this case, the fiber has been tapered such that the cladding has become the vacuum with $n_{2}=1$. Higher order modes can only be sustained if $V>V_{c} \simeq 2.405$, and below this value only the fundamental $\mathrm{HE}_{11}$ mode can propagate. The $V$ number can easily be controlled by choosing the fiber radius $[29,32]$.

Using cylindrical coordinates, the evanescent field around the nanofiber corresponding to the $\mathrm{HE}_{\ell m}$ mode with circular polarization is given by [37]

$$
\begin{gathered}
E_{r}=i C\left[(1-s) K_{\ell-1}(q r)+(1+s) K_{\ell+1}(q r)\right] e^{i(\omega t-\beta z)}, \\
E_{\phi}=-C\left[(1-s) K_{\ell-1}(q r)-(1+s) K_{\ell+1}(q r)\right] e^{i(\omega t-\beta z)}, \\
E_{z}=2 C(q / \beta) K_{\ell}(q r) e^{i(\omega t-\beta z)}
\end{gathered}
$$

where

$$
\begin{gathered}
s=\frac{1 / h^{2} a^{2}+1 / q^{2} a^{2}}{J_{\ell}^{\prime}(h a) /\left[h a J_{\ell}(h a)\right]+K_{\ell}^{\prime}(q a) /\left[q a K_{\ell}(q a)\right]}, \\
C=\frac{\beta}{2 q} \frac{J_{\ell}(h a) / K_{\ell}(q a)}{\sqrt{2 \pi a^{2}\left(n_{1}^{2} N_{1}+n_{2}^{2} N_{2}\right)}},
\end{gathered}
$$

and

$$
\begin{aligned}
N_{1}= & \frac{\beta^{2}}{4 h^{2}}\left[(1-s)^{2}\left[J_{\ell-1}^{2}(h a)+J_{\ell}^{2}(h a)\right]\right. \\
& \left.+(1+s)^{2}\left[J_{\ell+1}^{2}(h a)-J_{\ell}(h a) J_{\ell+2}(h a)\right]\right] \\
& +\frac{1}{2}\left[J_{\ell}^{2}(h a)-J_{\ell-1}(h a) J_{\ell+1}(h a)\right], \\
N_{2}= & \frac{J_{\ell}^{2}(h a)}{2 K_{\ell}^{2}(q a)}\left(\frac { \beta ^ { 2 } } { 4 q ^ { 2 } } \left[(1-s)^{2}\left[K_{\ell-1}^{2}(q a)-K_{\ell}^{2}(q a)\right]\right.\right. \\
- & \left.(1+s)^{2}\left[K_{\ell+1}^{2}(q a)-K_{\ell}(q a) K_{\ell+2}(q a)\right]\right] \\
- & \left.\frac{1}{2}\left[K_{\ell}^{2}(q a)+K_{\ell-1}(q a) K_{\ell+1}(q a)\right]\right) .
\end{aligned}
$$

The mode geometry is given by $J_{n}(x)$, the Bessel function of the first kind, $K_{n}(x)$, the modified Bessel function of the second kind, and $\beta$, the propagation constant of the fiber. The scaling factors are given by $q=\sqrt{\beta^{2}-n_{2}^{2} k_{0}^{2}}$ and $h=$ $\sqrt{n_{1}^{2} k_{0}^{2}-\beta^{2}}$, the normalization constant is $C$ and $s$ is a dimensionless parameter.

When the input light field is linearly polarized, it is convenient to write the cartesian components of the evanescent electric field as

$$
\begin{aligned}
E_{x}= & \sqrt{2} C\left[(1-s) K_{\ell-1}(q r) \cos \left(\phi_{0}\right)\right. \\
& \left.+(1+s) K_{\ell+1}(q r) \cos \left(2 \phi-\phi_{0}\right)\right] e^{i(\omega t-\beta z)},
\end{aligned}
$$

$$
\begin{aligned}
E_{y}= & \sqrt{2} C\left[(1-s) K_{\ell-1}(q r) \sin \left(\phi_{0}\right)\right. \\
& \left.+(1+s) K_{\ell+1}(q r) \sin \left(2 \phi-\phi_{0}\right)\right] e^{i(\omega t-\beta z)}, \\
E_{z}= & 2 \sqrt{2} i C(q / \beta) K_{\ell}(q r) \cos \left(\phi-\phi_{0}\right) e^{i(\omega t-\beta z)} .
\end{aligned}
$$

Here $\phi_{0}$ determines the orientation of polarization, with $\phi_{0}=$ 0 being along the $x$ axis and $\pi / 2$ being along the $y$ axis. The artificial vector potential produced by such evanescent fields around an optical nanofiber is then given by [35]

$$
\mathbf{A}=\hat{z} \hbar \kappa_{0}\left(n_{1}+1\right) \tilde{s}\left[\frac{\left|d_{r} E_{r}+d_{\phi} E_{\phi}+d_{z} E_{z}\right|^{2}}{1+\tilde{s}^{2}\left|d_{r} E_{r}+d_{\phi} E_{\phi}+d_{z} E_{z}\right|^{2}}\right],
$$

where $\tilde{s}=\frac{|\mathbf{d} \cdot \mathbf{E}|}{\hbar|\Delta|}$ and $d_{r}, d_{\phi}$, and $d_{z}$ are the components of the dipole moment $\mathbf{d}$ in cylindrical coordinates (see [35] for their detailed form). The corresponding magnetic field $\mathbf{B}=\nabla \times \mathbf{A}$ can be calculated to be

$$
\begin{aligned}
\mathbf{B}= & \frac{\hbar \kappa_{0} s^{2}\left(n_{1}+1\right)}{\left(1+\tilde{s}^{2}\left|d_{r} E_{r}+d_{\phi} E_{\phi}+d_{z} E_{z}\right|^{2}\right)^{2}} \\
& \times\left[\hat{\phi} \frac{\partial}{\partial r}\left|d_{r} E_{r}+d_{\phi} E_{\phi}+d_{z} E_{z}\right|^{2}\right. \\
& \left.-\hat{r} \frac{1}{r} \frac{\partial}{\partial \phi}\left|d_{r} E_{r}+d_{\phi} E_{\phi}+d_{z} E_{z}\right|^{2}\right] .
\end{aligned}
$$

This shows that the $\mathbf{B}$ field has only components in the $\hat{\phi}$ and $\hat{r}$ directions, which means that all field lines lie in the horizontal plane if the fiber is aligned along the vertical $\hat{z}$ direction.

For a BEC that is trapped cylindrically around a nanofiber, one can therefore expect to find vortex structures that wrap around the nanofiber and potentially close in on themselves in the form of vortex rings; however, depending on the exact form of the evanescent mode other structures are possible as well. Modulating the value of $\tilde{s}$ allows one to change the amplitude and range of the magnetic field and thereby change the size and shape of the generated vortex structures [35]. In the following we will focus on three different evanescent field configurations: the fundamental $\mathrm{HE}_{11}$ mode with circular polarization, the $\mathrm{HE}_{11}$ mode with linear polarization, and the $\mathrm{HE}_{21}$ mode with linear polarization. The electric field configurations and their corresponding artificial magnetic fields can be seen in Fig. 2. It is notable that using the circularly polarized fundamental $\mathrm{HE}_{11}$ mode leads to cylindrically symmetric electric [Fig. 2(a)] and artificial magnetic field configurations [Fig. 2(b)], whereas using linearly polarized light leads to a lobed structure for both quantities [see Figs. 2(c) and 2(d)]. When using linearly polarized light with the higher-order $\mathrm{HE}_{21}$ mode, an even more complex structure composed of four petals appears [see Figs. 2(e) and 2(f)] and the broken rotational symmetry suggests these fields will lead to the appearance of nonstandard flow excitations. While using even higher order modes or interfering different modes can lead to even more complicated fields [35], we concentrate here on the three examples above, as they demonstrate the large range of fundamental possibilities the system allows for.

\section{VORTEX CONFIGURATIONS}

To determine the vortex states that can be created by the evanescent fields around a nanofiber we solve the full 

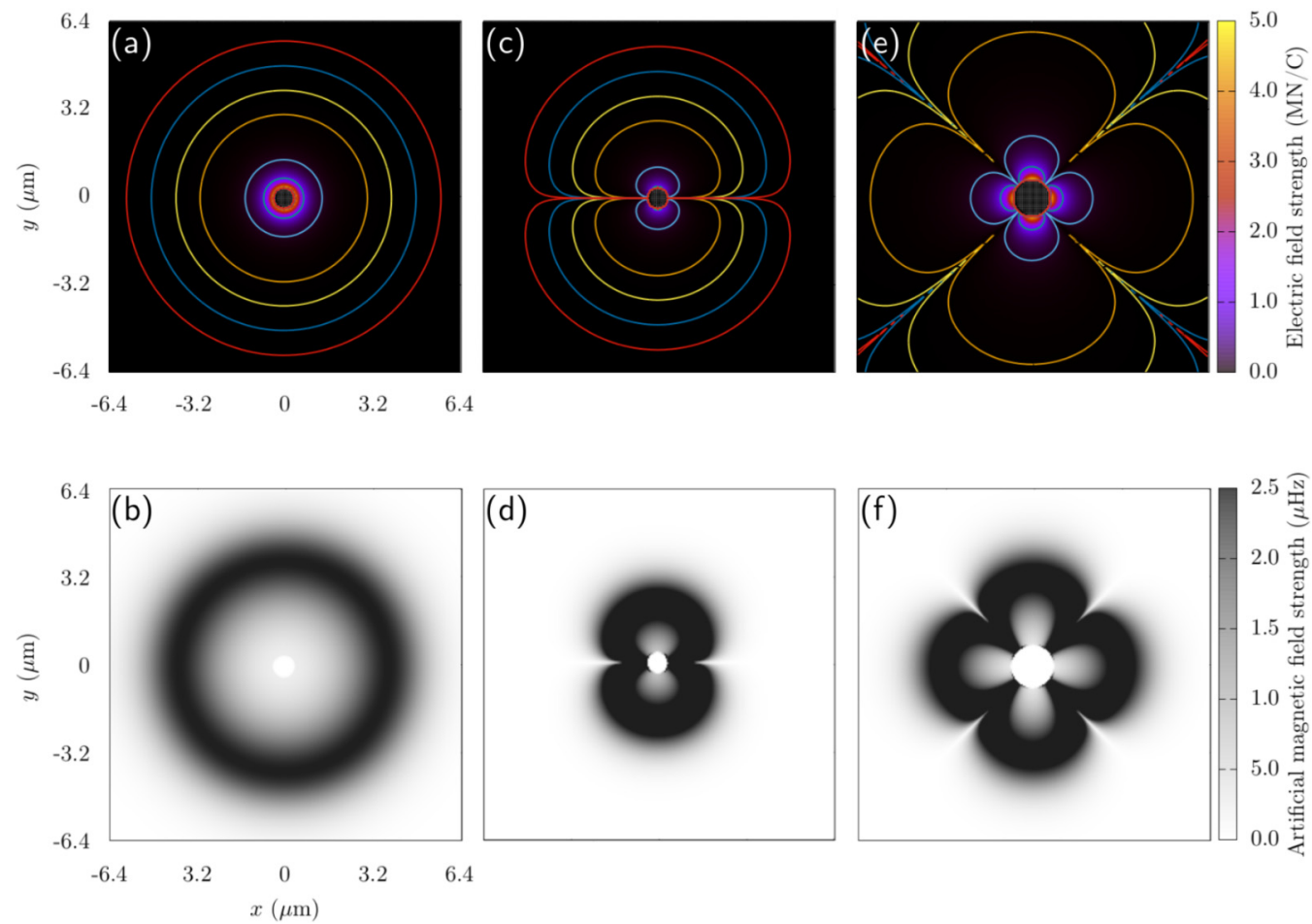

FIG. 2. Images of electric and artificial magnetic field profiles for [(a) and (b)] the fundamental $\mathrm{HE}_{11}$ mode with circular polarization, [(c) and (d)] the $\mathrm{HE}_{11}$ mode with linear polarization, and [(e) and (f)] the $\mathrm{HE}_{21}$ mode with linear polarization. For these calculations, the input power is $372 \mathrm{nW}$ in (a) and (b), $16 \mathrm{nW}$ in (c) and (d), and $418 \mathrm{nW}$ in (e) and (f). For the $\mathrm{HE}_{11}$ mode the nanofiber radius is $200 \mathrm{~nm}$ with blue-detuned light of $700 \mathrm{~nm}$, and for the $\mathrm{HE}_{21}$ mode the nanofiber radius is $400 \mathrm{~nm}$ with red-detuned light of $980 \mathrm{~nm}$.

three-dimensional Gross-Pitaevskii equation for a condensate trapped toroidally around the fiber. For this, we use the GPUE codebase [38] to describe a ${ }^{87} \mathrm{Rb}$ condensate with $1 \times 10^{5}$ atoms with a scattering length of $a_{s}=4.76 \times 10^{-9} \mathrm{~m}$ on a three-dimensional grid of $256^{3}$ points with a spatial resolution of $50 \mathrm{~nm}$. To clearly highlight the effects of the artificial magnetic fields, we assume a generic, external toroidal trapping around the fiber given by

$$
V=m\left[\omega_{r}^{2}(r-\eta)^{2}+\omega_{z}^{2} z^{2}\right],
$$

where we chose the trapping frequencies in the $r$ and $z$ directions to be $\omega_{r}=\omega_{z}=7071 \mathrm{~Hz}$ to match typical experimental conditions in fiber trapping [27]. The parameter $\eta$ defines the distance of the center of the toroidal condensate from the center of the fiber and is chosen such that the atoms are trapped outside the reach of the van der Waals potential of the fiber. For simulations of the $\mathrm{HE}_{11}$ mode, we assume a fiber radius of $200 \mathrm{~nm}$ and we use $\eta=3.20 \mu \mathrm{m}$ to create a toroidal BEC with an inner radius roughly $300 \mathrm{~nm}$ from the fiber surface. To simulate the effects of higher-order $\mathrm{HE}_{21}$ modes, we assume an increased fiber radius of $400 \mathrm{~nm}$, with all other parameters remaining the same. This creates a toroidal BEC with an inner radius roughly $150 \mathrm{~nm}$ from the fiber surface.

As a first example, we study the fundamental $\mathrm{HE}_{11}$ mode with circular polarization, which is perfectly azimuthally symmetric. One can therefore expect to find vortex lines that wrap around the fiber at a constant radius and reconnect onto themselves. This is confirmed in Fig. 3(a), where we show the equilibrium solution for a field strength that leads to exactly one vortex ring. For linearly polarized $\mathrm{HE}_{11}$ modes, the circular symmetry is broken and one can see from Fig. 3(c) that the vortex lines bend towards the inner edge of the condensate, creating two vortex lobes. This can be easily understood by realizing that the vortex lines have to follow lines of constant magnetic fields, which in these areas also bend towards and vanish into the fiber surface. However, this also means that the field lines come very close when approaching the surface, and careful examination of the condensate density shows that the vortex lines do not follow the field lines into the fiber surface, but rather connect to the neighboring lobe when they approach each other within a healing length. In fact, one can continuously go from the circular to the linear setting by considering elliptically polarized light, which leads to vortex rings that are deformed and interpolate between the azimuthally symmetric and fully folded in structure [see Fig. 3(b)]. Finally, for the linearly polarized $\mathrm{HE}_{21}$ mode, the superfluid system responds by creating vortex lines arranged in a four-petal shape, again mimicking the geometry of the artificial magnetic field [see Fig. 4]. For this situation we also show it is possible to create multiples of these vortex structures by increasing the field strength and that, for low densities of these structures, they arrange themselves on top of the maximum of the inhomogeneous $B$ field inside the condensate [see Fig. 5(b) for the $\mathrm{HE}_{11}$ mode].

To study control of multiple vortex structures with this system, we show that by increasing the $B$-field strength even further for the $\mathrm{HE}_{11}$ mode, we can create an even larger number of vortex rings, which at a certain density, make a transition to arranging themselves in a triangular geometry, forming the equivalent of the famous Abrikosov lattice [see 

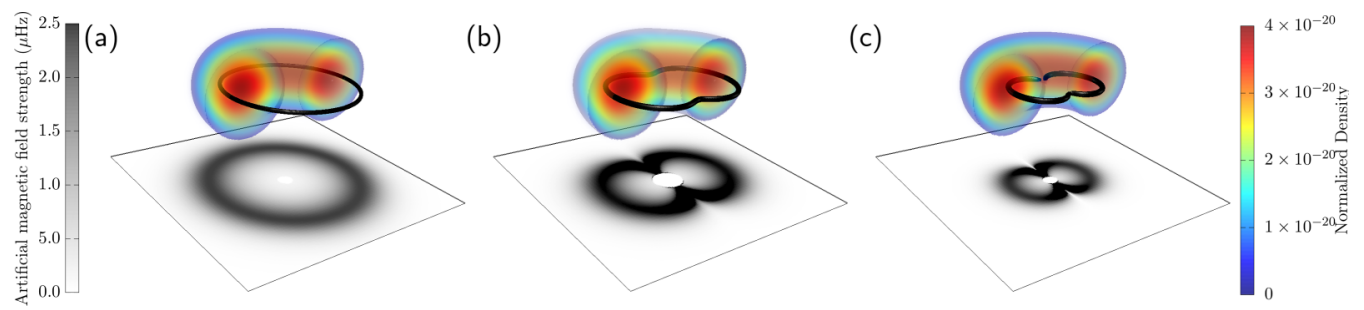

FIG. 3. Vortex configurations for different magnetic field profiles from the nanofiber for the fundamental HE 11 mode with (a) circular polarization, (b) elliptical polarization, and (c) linear polarization along the $\hat{y}$ direction. The vortex distributions have been found via an isosurface on the Sobel filtered wave function density for a ${ }^{87} \mathrm{Rb}$ BEC and all optical fiber fields are normalized and for a nanofiber of $200 \mathrm{~nm}$ in radius with blue-detuned light of $700 \mathrm{~nm}$. The magnetic field profiles shown in the shaded region beneath wavefunction density are similar to those in Fig. 2(b) and 2(d).

Figs. 5(a), 5(b), and 5(c)]. However, as the artificial magnetic field is strongly inhomogeneous, this lattices forms close to and around the maximum of the magnetic field.

It is therefore clear that one can control the shape of each vortex structure and their number by purely controlling the optical fields that are fed into the fiber, and that artificial magnetic fields around optical fibers provide unprecedented control for the creation of vortex ring-like structures. In fact, because all optical fields can also be time dependent, this system can potentially be used for studies of the dynamical properties of these rings; however, in the latter case, additional care needs to be taken as high magnetic field values change the potential geometry of the atoms in the BEC due to a coupling between the artificial vector potential $\mathbf{A}$ and the trapping potential $V$. In this case, the external potential $V$ gets modified by a term proportional to $\mathbf{A}^{2}$, which has an effect on the condensate density beyond exciting rotation. Time-dependent changes to the $\mathbf{A}$ field through changes in the laser intensity therefore also lead to phonon excitations, which in turn have an influence on the vortex line dynamics. However, studies of the response of the wave-function density to time-dependent artificial magnetic fields go beyond the scope of this work.

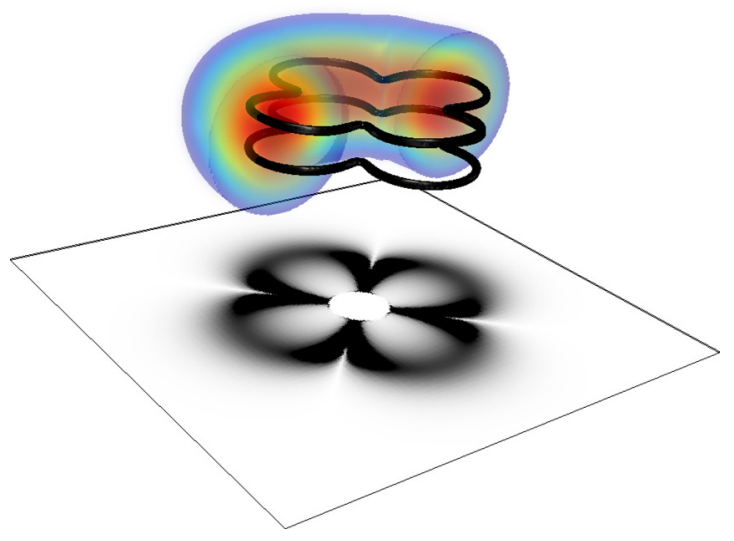

FIG. 4. Vortex configuration for the $\mathrm{HE}_{21}$ mode with linear polarization along the $\hat{y}$ direction. The vortex distributions have been found via an isosurface on the Sobel filtered wave function density for $\mathrm{a}{ }^{87} \mathrm{Rb} \mathrm{BEC}$ and the optical fiber fields are for a nanofiber of $400 \mathrm{~nm}$ in diameter for the bottom image with red-detuned light of $980 \mathrm{~nm}$. The magnetic field profile is similar to the one shown in Fig. 2(f), and has been calculated for a nanofiber of $400 \mathrm{~nm}$ radius with red-detuned light of $980 \mathrm{~nm}$.

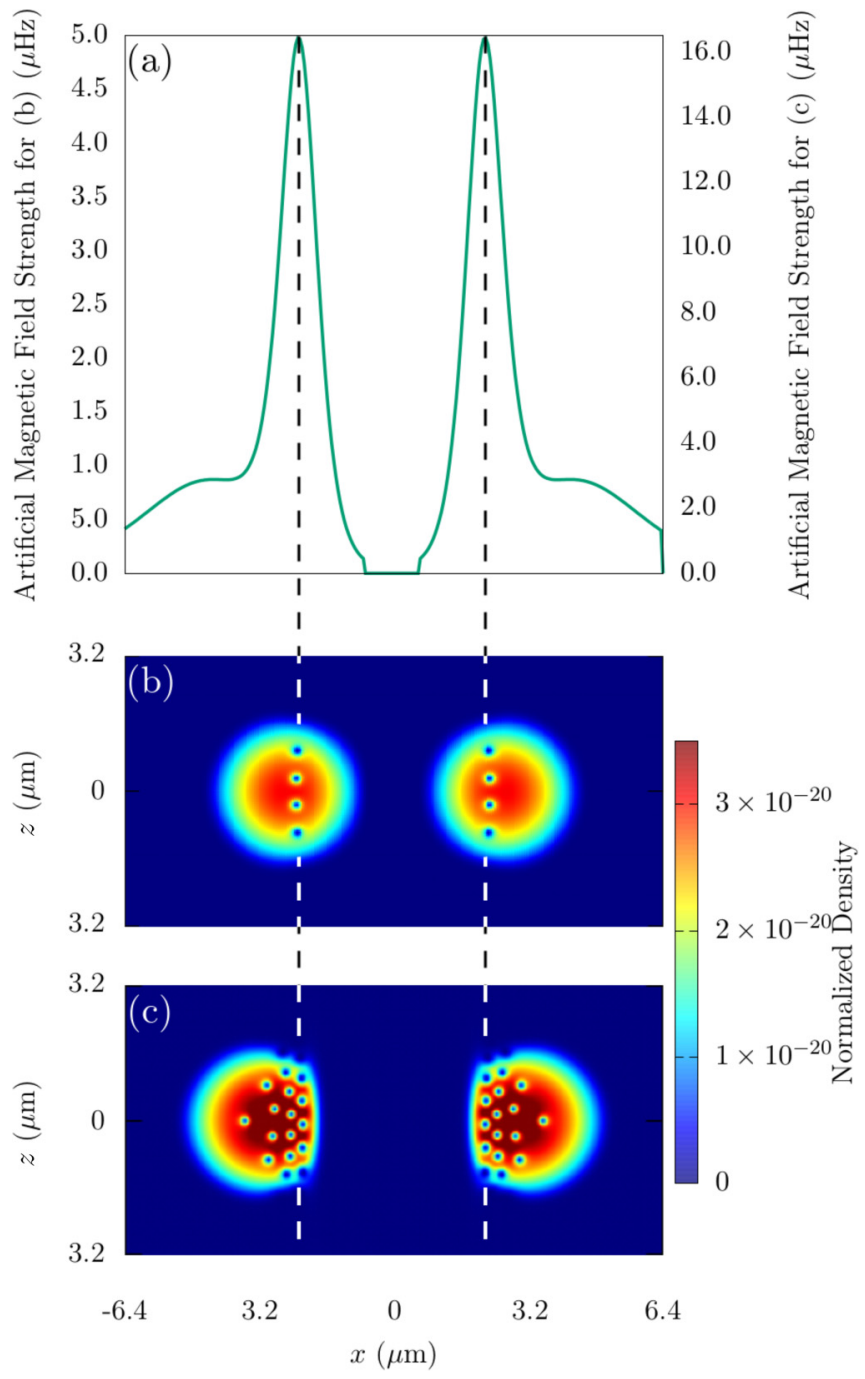

FIG. 5. (a) The magnetic field profile along the $x$ direction for the fundamental $\mathrm{HE}_{11}$ mode with circular polarization outside a fibre of $200 \mathrm{~nm}$ radius. Note that for this mode and polarization the whole system is azimuthally symmetric. For weak fields [see (b)] this leads to a small number of vortices that align along the line at which the magnetic field is maximal and for larger fields [see (c)] more vortex rings appear that form the beginning of an Abrikosov lattice. The optical fiber field and wave-function density have been normalized and are for a nanofiber of $200 \mathrm{~nm}$ in diameter with blue-detuned light of $700 \mathrm{~nm}$ and $\mathrm{a}^{87} \mathrm{Rb}$ BEC respectively. 
Nevertheless, let us stress, that for constant optical fields these (deformed) vortex-ring structures are stable and unique to creating vortex rings with artificial magnetic fields. They cannot be excited using simple rotation in singly connected potentials.

\section{DISCUSSION}

We have shown that it is possible to create and control vortex rings and more complicated vortex structures in three dimensions using the artificial magnetic field around an optical nanofiber. To the best of our knowledge, there is currently no other known method to generate the structures obtained from nonazimuthally symmetric modes from the linearly and elliptically polarized evanescent fields shown in Figs. 3(b), 3(c), and 4. Furthermore, using even higher-order modes or interference between different modes will allow creation of even more complicated vortex structures in a controlled way. In general, detecting the presence of vortex rings and more complicated structures in a three-dimensional BEC is a difficult problem, as absorption spectroscopy only provides a two-dimensional picture of an integrated density. However, due to the fiber necessitating a multiply connected geometry, the system presented here allows one to identify whether vortex structures are present by exciting a scissors- like mode of the condensate [39-41]. Different to the situation in simply connected potentials though, where an elliptical potential is rotated in one direction around its minimum, here an elliptical toroidal potential can be rotated inwards or outwards around the trapping minimum. As at any point along the azimuthal direction vortices only goes in one direction, this quench will lead to scissors-like oscillation, whose frequency will be influenced by the presence of vortex lines. We are currently exploring this aspect further.

Finally, being able to stably create these nontrivial vortex configurations may be the first step toward creating more complex structures like vortex knots in a single-component superfluid BEC system with the optical nanofiber; however, to generate these structures, the magnetic field must have a dependence on $\hat{z}$, which is not present in our current model.

\section{ACKNOWLEDGMENTS}

This work has been supported by the Okinawa Institute of Science and Technology Graduate University and used the computing resources of the Scientific Computing and Data Analysis section. This work has also been supported by Japanese Society for the Promotion of Science KAKENHI JP17J01488.
[1] C. J. Pethick and H. Smith, Bose-Einstein Condensation in Dilute Gases, 2nd ed. (Cambridge University Press, Cambridge, 2008).

[2] K. W. Madison, F. Chevy, W. Wohlleben, and J. Dalibard, Vortex Formation in a Stirred Bose-Einstein Condensate, Phys. Rev. Lett. 84, 806 (2000).

[3] J. R. Abo-Shaeer, C. Raman, J. M. Vogels, and W. Ketterle, Observation of vortex lattices in Bose-Einstein condensates, Science 292, 476 (2001).

[4] D. H. Wacks, A. W. Baggaley, and C. F. Barenghi, Large-scale superfluid vortex rings at nonzero temperatures, Phys. Rev. B 90, 224514 (2014).

[5] B. P. Anderson, P. C. Haljan, C. A. Regal, D. L. Feder, L. A. Collins, C. W. Clark, and E. A. Cornell, Watching Dark Solitons Decay into Vortex Rings in a Bose-Einstein Condensate, Phys. Rev. Lett. 86, 2926 (2001).

[6] A. Bulgac, M. McNeil Forbes, M. M. Kelley, K. J. Roche, and G. Wlazłowski, Quantized Superfluid Vortex Rings in the Unitary Fermi Gas, Phys. Rev. Lett. 112, 025301 (2014).

[7] M. J. H. Ku, B. Mukherjee, T. Yefsah, and M. W. Zwierlein, Cascade of Solitonic Excitations in a Superfluid Fermi Gas: From Planar Solitons to Vortex Rings and Lines, Phys. Rev. Lett. 116, 045304 (2016).

[8] M. R. Matthews, B. P. Anderson, P. C. Haljan, D. S. Hall, C. E. Wieman, and E. A. Cornell, Vortices in a Bose-Einstein Condensate, Phys. Rev. Lett. 83, 2498 (1999).

[9] T. Yefsah, A. T. Sommer, M. J. H. Ku, L. W. Cheuk, W. Ji, W. S. Bakr, and M. W. Zwierlein, Heavy solitons in a fermionic superfluid, Nature (London) 499, 426 (2013).

[10] A. A. Abrikosov, The magnetic properties of superconducting alloys, J. Phys. Chem. Solids 2, 199 (1957).
[11] M. Tsubota, Turbulence in quantum fluids, J. Stat. Mech. Theor. Exp. (2014) P02013.

[12] C. F. Barenghi, L. Skrbek, and K. R. Sreenivasan, Introduction to quantum turbulence, Proc. Natl. Acad. Sci. U.S.A. 111, 4647 (2014).

[13] S. W. Seo, B. Ko, J. H. Kim, and Y. Shin, Observation of vortexantivortex pairing in decaying $2 \mathrm{D}$ turbulence of a superfluid gas, Sci. Rep. 7, 4587 (2017).

[14] N. Navon, A. L. Gaunt, R. P. Smith, and Z. Hadzibabic, Emergence of a turbulent cascade in a quantum gas, Nature (London) 539, 72 (2016).

[15] J. Ruostekoski and J. R. Anglin, Creating Vortex Rings and Three-Dimensional Skyrmions in Bose-Einstein Condensates, Phys. Rev. Lett. 86, 3934 (2001).

[16] I. Shomroni, E. Lahoud, S. Levy, and J. Steinhauer, Evidence for an oscillating soliton/vortex ring by density engineering of a Bose-Einstein condensate, Nat. Phys. 5, 193 (2009).

[17] J. Ruostekoski and Z. Dutton, Engineering vortex rings and systems for controlled studies of vortex interactions in BoseEinstein condensates, Phys. Rev. A 72, 063626 (2005).

[18] N. S. Ginsberg, J. Brand, and L. V. Hau, Observation of Hybrid Soliton Vortex-Ring Structures in Bose-Einstein Condensates, Phys. Rev. Lett. 94, 040403 (2005).

[19] B. Jackson, J. F. McCann, and C. S. Adams, Vortex line and ring dynamics in trapped Bose-Einstein condensates, Phys. Rev. A 61, 013604 (1999).

[20] F. Pinsker, N. G. Berloff, and V. M. Pérez-García, Nonlinear quantum piston for the controlled generation of vortex rings and soliton trains, Phys. Rev. A 87, 053624 (2013).

[21] M. Abad, M. Guilleumas, R. Mayol, and M. Pi, Vortex rings in toroidal Bose-Einstein condensates, Laser Phys. 18, 648 (2008). 
[22] J. Dalibard, F. Gerbier, G. Juzeliūnas, and P. Öhberg, Colloquium: Artificial gauge potentials for neutral atoms, Rev. Mod. Phys. 83, 1523 (2011).

[23] M. Mochol and K. Sacha, Artificial magnetic field induced by an evanescent wave, Sci. Rep. 5, 7672 (2015).

[24] J. M. Ward, D. G. O'Shea, B. J. Shortt, M. J. Morrissey, K. Deasy, and S. G. Nic Chormaic, Heat-and-pull rig for fiber taper fabrication, Rev. Sci. Instrum. 77, 083105 (2006).

[25] L. Tong, R. R. Gattass, J. B. Ashcom, S. He, J. Lou, M. Shen, I. Maxwell, and E. Mazur, Subwavelength-diameter silica wires for low-loss optical wave guiding, Nature (London) 426, 816 (2003).

[26] A. Yariv, Introduction to Optical Electronics (Holt, Rinehart and Winston, New York, 1976).

[27] E. Vetsch, D. Reitz, G. Sagué, R. Schmidt, S. T. Dawkins, and A. Rauschenbeutel, Optical Interface Created by Laser-Cooled Atoms Trapped in the Evanescent Field Surrounding an Optical Nanofiber, Phys. Rev. Lett. 104, 203603 (2010).

[28] C. Lacroûte, K. S. Choi, A. Goban, D. J. Alton, D. Ding, N. P. Stern, and H. J. Kimble, A state-insensitive, compensated nanofiber trap, New J. Phys. 14, 023056 (2012).

[29] T. Nieddu, V. Gokhroo, and S. Nic Chormaic, Optical nanofibres and neutral atoms, J. Opt. 18, 053001 (2016).

[30] G. Sagué, E. Vetsch, W. Alt, D. Meschede, and A. Rauschenbeutel, Cold-Atom Physics Using Ultrathin Optical Fibers: Light-Induced Dipole Forces and Surface Interactions, Phys. Rev. Lett. 99, 163602 (2007).

[31] L. Russell, K. Deasy, M. J. Daly, M. J. Morrissey, and S. Nic Chormaic, Sub-Doppler temperature measurements of lasercooled atoms using optical nanofibres, Meas. Sci. Technol. 23, 015201 (2011).
[32] R. Kumar, V. Gokhroo, K. Deasy, A. Maimaiti, M. Frawley, C. Phelan, and S. Nic Chormaic, Interaction of laser-cooled ${ }^{87} \mathrm{Rb}$ atoms with higher order modes of an optical nanofibre, New J. Phys. 17, 013026 (2015).

[33] F. Le Kien, V. I. Balykin, and K. Hakuta, Atom trap and waveguide using a two-color evanescent light field around a subwavelength-diameter optical fiber, Phys. Rev. A 70, 063403 (2004).

[34] C. F. Phelan, T. Hennessy, and Th. Busch, Shaping the evanescent field of optical nanofibers for cold atom trapping, Opt. Express 21, 27093 (2013).

[35] R. Sachdeva and Th. Busch, Creating superfluid vortex rings in artificial magnetic fields, Phys. Rev. A 95, 033615 (2017).

[36] M. Cheneau, S. P. Rath, T. Yefsah, K. J. Günter, G. Juzeliunas, and J. Dalibard, Geometric potentials in quantum optics: A semi-classical interpretation, Europhys. Lett. 83, 60001 (2008).

[37] V. G. Minogin and S. Nic Chormaic, Manifestation of the van der Waals surface interaction in the spontaneous emission of atoms into an optical nanofiber, Laser Phys. 20, 32 (2010).

[38] J. Schloss and L. J. O'Riordan, GPUE: Graphics Processing Unit Gross-Pitaevskii Equation solver, J. Open Source Softw. 3, 1037 (2018).

[39] M. Cozzini, S. Stringari, V. Bretin, P. Rosenbusch, and J. Dalibard, Scissors mode of a rotating Bose-Einstein condensate, Phys. Rev. A 67, 021602 (2003).

[40] D. Guéry-Odelin and S. Stringari, Scissors Mode and Superfluidity of a Trapped Bose-Einstein Condensed Gas, Phys. Rev. Lett. 83, 4452 (1999).

[41] O. M. Marago, S. A. Hopkins, J. Arlt, E. Hodby, G. Hechenblaikner, and C. J. Foot, Observation of the Scissors Mode and Evidence for Superfluidity of a Trapped BoseEinstein Condensed Gas, Phys. Rev. Lett. 84, 2056 (2000). 